\title{
Cognitive Emotional Regulation: In the Context of Breast Cancer
}

\author{
Ms. Tina Fernandes ${ }^{1}$, Ms. Aarthi R. ${ }^{2}$, Ms. Sakshi Bohra ${ }^{3}$
}

\section{ABSTRACT}

Internal resources are likely to have great brunt on embracing different type of coping strategies among those who have encountered negative experiences/events in life like diagnosed with cancer. Among these coping strategies is cognitive emotion regulation. There were 44 breast cancer patients selected as sample of study from the cosmopolitan city of Hyderabad. The objective of the study was to determine if there was a difference between younger and older women suffering with breast cancer undergoing treatment with respect to cognitive emotion regulation. To determine if there is a difference between employed and not employed women suffering with breast cancer undergoing treatment with respect to cognitive emotion regulation . The instrument used in the study is cognitive emotion regulation questionnaire Analysis of the obtained results shows that there was significant difference between younger and older women with cancer, with reference to the dimension of rumination. There was significant differences between employed and unemployed women suffering with breast cancer undergoing treatment with respect to only focus on planning. This would propose modifications in intervention studies and tailor make them according to stressors instead of personal variable of age and consider their role behaviors related to their occupational status.

Keywords: negative life events, cognitive emotional regulation, breast cancer.

Human beings position themselves on various stages of the continuum of wellbeing i.e. between health and illness. Given the nature of illness, unlike the early $19^{\text {th }}$ century when the communicable diseases created menace, in the present scenario the debacle of non communicable diseases is prominent. One such non communicable disease which is claiming several lives worldwide is 'cancer'. At present, the second leading disease is cancer which leads to death in most of the cases. According to National Cancer Institute, "cancer is a term used for diseases in which abnormal cells divide without control and are able to invade other tissues." Cancer is a disease caused by an uncontrollable division of abnormal cell in a part of the body.

${ }^{1}$ Head, Dept of Psychology, St Francis College for Women

${ }^{2}$ Research Scholar, Centre for Health Psychology, University of Hyderabad

${ }^{3}$ Psychology Graduate, Dept of Psychology, St Francis College for Women

(C) 2015 I T Fernades, Aarthi, S Bohra; licensee IJIP. This is an Open Access Research distributed under the terms of the Creative Commons Attribution License (http://creativecommons.org/licenses/by/2.0), which permits unrestricted use, distribution, and reproduction in any Medium, provided the original work is properly cited. 


\section{Cognitive Emotional Regulation: In the Context of Breast Cancer}

Cancer is not just one disease but have a number of diseases under its broad heading and usually are named using the name of the organ which is affected for example( lung cancer, breast cancer ,colon cancer). The National Cancer Institute has broadly classified cancer into five types. They are carnimona, sarcoma, leukemia, lymphoma and myeroma, central nervous system cancer.

There are various types of cancer but the diseases begin from a single cell which is the primary unit in the body and form a mass which is known as tumor. Cancer figures among the leading cause of death worldwide, accounting for 8.2 million deaths in 2012 (Globocan 2012, IARC). According to American Cancer Society there are 76 types in cancer and the second most common is breast cancer. National Cancer Institute defines breast cancer as a type of cancer that forms in the breast tissues and has mainly three types in which the most common one is ductual carcinoma which embark from the lining of milk ducts which carry milk from the lobules of the breast to the nipple. The next type of breast cancer is lobular carcinoma which affects the lobules and the last type of breast cancer is invasive breast cancer in this type the cancer spreads from the lobules to the surrounding normal tissues. It is one of the most common cancers in women and is rapidly increasing. It has been reported that every year over 1.15 million women worldwide are diagnosed with breast cancer and 502,000 die from this diseases (WHO, 2014).

There are many changes in the body with breast cancer both the major noticeable change is a lump or mass that feels different from the other breast tissues. $80 \%$ of the breast cancers are out in the open when women feel this lump (Merck manual of diagnosis therapy 2005). There can be other signs also rather than the lump like rashes on the breast, the nipple becomes inverted, skin dimpling, change in skins color or the texture of the skin may also change or discharge of clear or bloody fluid from the nipple (Watson 2008). There are some other symptoms also like itching, pain, redness etc. The key risk factor of breast cancer is women and old age (Reeder \& Vogel 2008). There are some other factors like overweight, drinking alcohol, using hormone replacement theory, taking birth control pills, not having children, or having children after 35 years of age, not breast feeding children and so on. In most of the women, breast cancer leads to death.

Cancer is a disease that spreads to the different parts of the body and has basically four stages. According to the American Cancer Society (2014) staging is “the severity of a person's cancer based on the size and/or extend (reach) of the original (primary) tumor and whether or not cancer has spread in the body." though there are even men who are diagnosed with breast cancer but their number when compared to women is very less. Breast cancer is an extremely stressful event and often is accompanied by the experience of distressing symptoms and psychological immobility (Cella, Mahon \& Donovan, 1990). This disease not only affects physically but also affects psychologically. Individuals with breast cancer have lot of problems in coping with the disease they also have anxiety and some cancer survivors also go in depression. 


\section{Cognitive Emotional Regulation: In the Context of Breast Cancer}

Detection of cancer and living with cancer and undergoing the treatment is stressful for the individuals and their care takers. Given such a stressful life event this beckons coping specifically adaptive coping. However, maladaptive coping like denial is found high in cancer patients. It is important that adaptive coping which includes both cognitive and emotion coping strategies is effective in coping with stressful events like cancer.

Emotions are strong elements that influence the individual's decision making, their daily activities and eventually their behaviors. "An emotion is a complex psychological state that involves three distinct components: a subjective experience, a physiological response, and a behavioral or expressive response." (Hockenbury \& Hockenbury, 2007 ). Emotion is a subjective, conscious experience distinguished primarily by psycho physiological expressions biological reactions and mental states. Emotions to a very great extent regulates our lives. Emotions have the capacity to influence our decision making process. They interact with our mood, character, and personality. Emotions can be classified in many ways. Exkman (1972) put forward six basic emotions which are fear, disgust, anger, surprise, happiness, and sadness. However, the term "emotion" is hard to define and even more rigid to understand completely. The experiences of emotions are dynamic; the interplay between emotions takes place when one fades and the other becomes prominent. Given such a dynamic nature of emotions, there requires the ability to regulate one's emotions timely and appropriately.

"Emotion regulation is a conscious or unconscious control of emotion, mood or affect" (Van der Wal \& Kowalczyk, 2013). Emotional regulation refers to all the strategies that are used to reduce, increase, or maintain emotion (Gross, 2001). It is a process through which human beings sort out or filter their emotions. Emotions vary from culture to culture and also from situation to situation. Therefore this emotion regulation is also connected with the culture and situation of the individual. Gross (1998) defines the ideas of emotional regulation as "the process by which individuals influence which emotion they have, when they have them, and how they express these emotions." As stated by this definition individuals have complete control over their emotions and can regulate their emotions consciously as well as unconsciously. Emotion regulation has both positive as well as negative outcomes. How a person regulates his/her emotions describes his/her ability to relate and regulate the social interaction. Emotion regulation is in different levels in different individual. Emotion regulation largely depends on emotional intelligence. Through emotional intelligence one can identify and manage their own emotions as well as others emotions. Emotion regulation is presumed to be a major factor in determining well being and/or successful functioning (Cicchetti, Ackerman \& Izard, 1995; Thompson, 1991). The effort to regulate one's emotions requires the role of cognition. The reason being the effect of emotions can be overwhelming and at times hindering daily functioning. Thus, cognition helps in understanding the emotion, cope with it and regulate it.

Cognitive and biological approaches to psychology have often been used in understanding how we experience emotions. The word 'cognitive coping' is used interchangeably with the term 


\section{Cognitive Emotional Regulation: In the Context of Breast Cancer}

'cognitive emotion regulation'. Both these concepts can be understood as a cognitive way of organizing the intake of emotionally exciting information (Thompson, 1991). "Cognitive emotion regulation refers to the conscious, cognitive way of handling the intake of emotionally arousing information” (Garnefski, Raaiji, \& Spinhoven, 2001; Thompson, 1991). Cognitive emotion regulation is a process in which we regulate our emotions by either changing the way we think about a particular situation or by satisfying the demand which the situation poses and alters the emotional impact. It is a part of cognitive coping and falls under the broader concept emotional regulation. (Gross, 1999; Thompson, 1994) defines cognitive emotion regulation as "all the extrinsic and intrinsic processes responsible for monitoring, evaluating, and modifying emotional reactions, especially their intensive and temporal features”. Cognitive emotion regulation or cognitive reappraisal is a coping strategy but very different strategy. Like other strategies' it does not deal with the environment or with the physical and emotional response but concentrates in an individual's perception of a situation.

It is appropriate to be studied in the situation like cancer where instead of external factors, the individual's perception is the most appropriate to managed healthily.

Thus, it is important that to cope with life stressors like living with cancer there needs to assess their coping in terms of adaptive coping strategies like cognitive emotional regulation. The coping style and strategies tend to age as age progresses, since maturity may play a role in their perception. In addition to age, occupational status of being employed or unemployed plays an important role. As in the former, fitness to get to work can pose an additional threat and the case of latter, financial dependency on the other earning family members is a cause of concern. Since there is paucity of studies in such realm, it requires to be further explored, which is the objective of the present study.

\section{OBJECTIVES}

a) To determine if there is a difference between younger and older women suffering with breast cancer undergoing treatment with respect to cognitive emotion regulation.

b) To determine if there is a difference between employed and unemployed women suffering with breast cancer undergoing treatment with respect to cognitive emotion regulation.

\section{METHOD}

\section{Design}

The present research is a quantitative study and has adopted a between group design to assess the differences in cognitive emotion regulation and its 9 dimensions (viz., acceptance, self blame, positive refocusing, positive reappraisal, putting into perspective, rumination, catastrophizing, and other blame) 44 women with breast cancer patients residing in the metropolitan city of Hyderabad . 


\section{Participants}

A purposive sampling method was used to collect the sample of 44 women suffering from breast cancer undergoing treatment. The sample was divided into two age groups and the patients were between the age group of $(\mathrm{N}=26)$ 45-55 years and $(\mathrm{N}=16)$ 60-70 years from the metropolitan city of Hyderabad. There were 22 employed and 22 unemployed. Participants with $2^{\text {nd }}$ and $3^{\text {rd }}$ stage were included. Patients with $4^{\text {th }}$ stage breast cancer were excluded

\section{Instruments}

Cognitive emotion regulation questionnaire (CERQ) a standardized questionnaire is used in this study which measures cognitive coping strategies after negative events or experiences. The questionnaire has been developed by Garnfski, Raaiji \& Spinhoven (2001). It uses a liketscale with 5 answer - choices which were almost never, sometimes, regularly, often, almost always It is a multi-dimensional questionnaire. It makes a distinction between peoples thoughts and real actions. It consists of 9 subscales and 36 items and is a self - report questionnaire. Rumination, self-blame, acceptance, positive refocusing, blaming others, refocus on planning, positive reappraisal, putting into perspective, and catastrophizing. The questionnaire has good factorial validity and high reliabilities, with Cronbach's $\alpha$ s ranging between .75 and .85 .

\section{Procedure}

At the outset, hospitals were selected and permission was sought from the management of the respective hospitals to recruit the participants. Participants i.e. the breast cancer patients undergoing treatment were approach for study. Informed .consent was taken from the participants prior to the administration of questionnaire. 


\section{RESULTS}

To analyze the results of the present study descriptive statistics like Mean and SD and inferential statistics like t test were used.

Table 1: Demographic details of the women suffering with breast cancer and undergoing treatment.

NO Characteristics N

1.

Sex

Females

44

2.

Education

Illiterates

4

School

22

Intermediate

5

Graduates

9

Post Graduates

3

3.

Occupation

Employed

22

Unemployed

22

4.

\section{Age Group}

45-55

26

60-70

18

5.

Marital Status

Married

44

Unmarried

0 
Table 2 Mean, Standard deviation and t-ratio of women suffering from breast cancer and undergoing treatment divided into two age groups.

Age

group

$\begin{array}{llll}\text { Variables } & 45-54 & 60-69 & \mathrm{t}(42)\end{array}$

$\begin{array}{llll}M & \text { SD } & \text { M } & \text { SD }\end{array}$

Self blame

9.69

3.52

9

3.41

0.65

Acceptance

12.15

3.06

12.06

2.82

0.99

Rumination

11.88

2.63

$10.17 \quad 2.64$

2.12*

Refocus on planning

10.5

3.02

11.83

2.28

1.58

Positive refocussing

11.69

4.25

11.67

3.96

0.02

Positive reappraisal

12.38

4.42

13.28

3.27

0.73

Putting into perspective

12.19

3.21

12.17

4.13

0.02

Catastrophizing

11.73

3.79

9.56

3.11

2.00

Other blame

7.35

3.52

6.78

3.67

0.52

Note.*:p>0.05

The results indicate that mean, standard deviation and t ratio of Cognitive emotion regulation of Women with breast cancer between the two groups is not significant apart from rumination $(\mathrm{t}(42)=2.12, \mathrm{p}<0.01)$ which illustrate significant difference between the two groups. 
Table 3 Mean, Standard deviation and t-ratio of women with breast cancer undergoing treatment divided into employed and unemployed.

\begin{tabular}{llllll}
\hline Variables & \multicolumn{2}{l}{ Occupation } & & \\
& employed & \multicolumn{2}{l}{ unemployed } & $\mathrm{t}(42)$ \\
& $\mathrm{M}$ & $\mathrm{SD}$ & $\mathrm{M}$ & $\mathrm{SD}$ & \\
& & & & & \\
\hline Self blame & 9.61 & 4.02 & 11 & 2.94 & 1.31 \\
Acceptance & 12.22 & 2.88 & 12.04 & 2.9 & 0.24 \\
Rumination & 11.83 & 2.67 & 10.73 & 2.64 & 1.37 \\
Refocus on planning & 12 & 2.62 & 10.38 & 2.66 & $2.04^{*}$ \\
Positive refocussing & 11.89 & 3.97 & 11.54 & 4.08 & 0.34 \\
Positive reappraisal & 13.39 & 3.73 & 12.31 & 4 & 0.93 \\
Putting into perspective & 13.22 & 3.47 & 11.46 & 3.39 & 1.70 \\
Catastrophizing & 11.11 & 3.03 & 10.65 & 3.95 & 0.43 \\
Other blame & 7.89 & 3.97 & 6.58 & 3.04 & 1.23 \\
\hline Note.*:p $>0.05$ & & & & &
\end{tabular}

The results in the above table reveal that there is a significant difference between the employed and unemployed women suffering with breast cancer and undergoing treatment with respect to refocusing on planning $(\mathrm{t}(42)=2.04, \mathrm{p}<0.05)$ when compared to other dimensions which are not significant. 
Table 4: showing the correlation within the 9 dimensions (i.e. Rumination, self-blame, acceptance, positive refocusing, blaming others, refocus on planning, positive reappraisal, putting into perspective, and catastrophizing) of cognitive emotion regulation

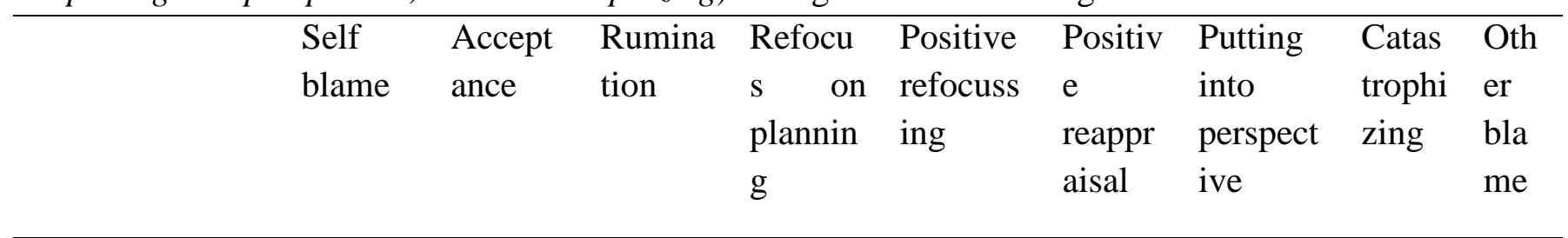

Self blame $\quad 1.00$

Acceptance $\quad 0.38^{*} \quad 1.00$

Rumination $\quad 0.29 \quad 0.44^{* *} \quad 1.00$

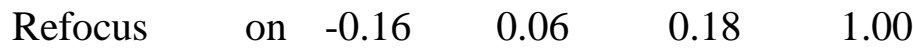

planning

$\begin{array}{llllll}\text { Positive } & -0.10 & -0.24 & 0.13 & 0.26 & 1.00\end{array}$

refocussing

$\begin{array}{lllllll}\text { Positive } & -0.19 & -0.17 & 0.03 & 0.34 * & 0.46 * * & 1.00\end{array}$

reappraisal

$\begin{array}{llllllll}\text { Putting into } & 0.09 & 0.26 & 0.25 & 0.48^{* *} & 0.03 & 0.17 & 1.00\end{array}$

perspective

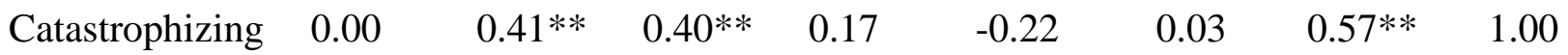

$\begin{array}{llllllllll}\text { Other blame } & -0.30 & 0.07 & -0.07 & 0.27 & -0.34 & 0.01 & 0.29 & 0.42 * & 1.0\end{array}$

Note. ${ }^{*}: \mathrm{p}>0.05 . * *: p>0.01$

Analysis of the obtained results illustrate that there are significant positive correlation within the dimensions of cognitive emotion regulation. There is a significant correlation between self-blame and acceptance $(r=0.38)$, acceptance and rumination $(r=0.44)$, refocus on planning and positive reappraisal ( $(\mathrm{r}=0.34)$, positive refocusing and positive reappraisal $(\mathrm{r}=0.46)$, refocus on planning and putting into perspective $(r=0.48)$, acceptance and catastrophizing $(r=0.41)$, rumination and catastrophizing $(\mathrm{r}=0.40)$, putting into perspective and catastrophizing $(\mathrm{r}=$ $0.57)$, and catastrophizing and other blame $(r=0.42)$. 


\section{DISCUSSION}

The purpose of the study was to investigate whether there were significant differences between the younger and older women suffering from breast cancer and undergoing treatment with respect to cognitive emotion regulation. The second objective of the study was whether there were significant differences between the employed and unemployed women suffering with breast cancer undergoing treatment with respect to cognitive emotion regulation.

Analysis of the obtained results shows that there are no significant differences between the mean score of younger and older women suffering from breast cancer undergoing treatment with respect to cognitive emotion regulation except for rumination. There was significant difference between younger and older women with cancer, with reference to the dimension of rumination. Rumination was found to be high in younger women than older women with cancer. This finding is consistent with the study that stated that older people have less ruminative thinking than other younger age groups (Sütterlin, Paap, Babic, Kübler, \& Vögele, 2012).

Except for the dimension of rumination, with reference cognitive emotional regulation there were no age related difference. Which may imply that age is playing a minimal role in coping with stressor. It also implies that having a common stressor-cancer, age related coping strategies are replaced with stressor related coping strategies.

The results also reveals that there was significant differences between employed and unemployed women suffering with breast cancer undergoing treatment with respect to only focus on planning. Focus on planning was found to be high in employed women when compared to unemployed women. This implies that working women experience the exposure to planning in their work settings. Such planning that includes both shot term and long term planning and the strategic planning according to one's assets and liabilities.

The findings also revealed that older and younger women mostly used the adaptive techniques of coping strategies like acceptance, positive reappraisal, putting into perspective and rarely used the non adaptive techniques like self blame. Both the groups did not use the technique of others blame which classically is a non adaptive technique similar results were found in a research study done on hardiness and optimism as moderators of the types of cognitive emotion regulation strategies among adolescents who had encountered negative life events (Subranaian \&Nithyanandan 2008). Similar results were found in a study done on breast cancer (Wrang, Yo, He, Che, Li, Yang \& Shu 2013).

A research study on psychological coping strategies in cancer patients shows that though there are few studies on alliance between coping strategies and cancer commencement but psychological intervention has a significant effect on the quality of lives of cancer patients (Sparch \& Sostaric, 2004). Another research study done on how do cancer patients manage unattainable personal goals and their emotions showed that reorganize meaningful goals and 
focusing on pleasant issues and psychological intervention were associated with more positive affect (Schroevers, Kraaji \& Grarnefski, 2008).

Analysis of the obtained results showed that women who were employed mostly tend to blame themselves when compared to the unemployed women. The mean of adaptive techniques like refocusing on planning, positive reappraisal and putting into perspective were higher in employed women when compared to unemployed women. Both the groups did not use other blame technique as copying technique which is typically a non adaptive technique.

Results also revealed that there was significant correlation in some dimension of cognitive emotion regulation. There was a significant correlation between acceptance and self blame, rumination and self blame, rumination and acceptance, positive reappraisal and refocusing on planning, positive reappraisal and positive refocusing, putting into perspective and refocusing on planning, catastrophizing and acceptance, catastropizing and rumination, catastropizing and putting into perspective, other blame and putting into perspective, and other blame and catastropizing. The intercorrelation implied the internal consistency of the tool when used for Indian population.

Cognitive emotion regulation appears to play an important role in cancer patient's psychological well-being. Implementing appropriate intervention during the early stages of treatment can build adequate adapting coping strategies in women with breast cancer which will have a positive affect on their treatment. Such interventions should be tailor made, keeping in mind the stressor instead of personal variables like age. Because the present study findings state that age related difference is not significant to the coping strategies used. In addition, such interventions should consider the occupational status of the individuals too. For better wellbeing of cancer patients, steps must be taken for them to adhere to treatment regimen, persevere the treatment procedure, and maintain one's adaptive coping and regulate their emotions cognitively.

\section{REFERENCES}

American Cancer $\quad$ Society $\quad$ (2014) from http://www.cancer.org/research/cancerfactsstatistics/cancerfactsfigures2014/

Cella, D. F, Mahon, S. M, Donovan, M. I. (1990). Cancer recurrence as a traumatic event. Behavioural Medicine, 16(1), 15-22.

Cicchetti, D., Ackerman, B. P ., \& Izard, C. R. (1995). Emotion and emotion regulation in developmental psychopathology. Development and Psychopathology, 7, 1-10

Ekman, P. (ed). (1972). Darwin and facial expression: a century of research in review. New York: Academic press: Academic press

Extramera \& Rey (2014) Health-related quality of life and emotion regulation strategies in the unemployed: a cross- sectional survey.

Garenfski, N. \& Kraaiji, V. K., Sponshoven, P. (2001). Negative life events, cognitive emotion regulation problems. Personality and individual differences. 30, 1311-1327. 
Globacon (2012) IARC. Cancer incidence and mortality worldwide. Retrieved from http://globocan.iarc.fr/Default.aspx

Gross, J.J. (1998). Emerging field of Emotion regulation: An Integrative Review. Review of General Psychology, 2(3), 214-219

Gross, J. J (2001). Emotion regulation in adulthood: timing is everything. Current directions in psychological science $10(6) 214-219$.

Hockenbury, D. H. \& Hockenbury, S. E. (2007). Discovering psychology. New York: Worth Publishers.

Merck manual of diagnosis therapy (2005). Retrieved from http://www.merckmanuals.com/professional

National Cancer Institute. Retrieved from http://www.cancer.gov/

Reeder, J. G. \& Vogel, V. G. (2008). Breast cancer management, Second edition.

Schroevers, M., Kraaij, V. \& Garnefski, N. (2008). How do cancer patients manage unattainable personal goals and regulate their emotions? An examination of the relations between goal adjustment, cognitive emotion-regulation strategies, and positive and negative affect. British Journal of Health Psychology, 13, 551-62

Sprah, L. \& Sostaric, M. (2004) Coping Strategies in cancer patients. Radiological Oncology, 38(1), 35-42.

Subramanian, S. \& Nithyanandan, D. V. (2008). Hardiness and optimism as moderators of the type of cognitive emotion regulation strategies among adolescents who had encountered negative life events. Journal of Indian Health Psychology, 2(2), 167-177

Sütterlin, S., Paap, M. C. S., Babic, S., Kübler, A. \& Vögele, C. (2012). Rumination and Age: Some Things Get Better. Journal of Aging Research, 2012. doi:http://dx.doi.org/10.1155/2012/267327

Thompson, R.A. (1991). Emotion regulation and Emotion development. Educational psychology review, 3, 269-307.

Thompson, R.A. (1991). Emotion regulation: A theme in search for definition. Monographs of society for research in children development, 59, 25-52

Van der Wal, C. N., \& Kowalczyk, W. (2013). Detecting changing emotions in human speech by machine and humans. Applied intelligence, 39(4), 675-691

Watson, M. (2008). Breast cancer research. Retrieved from http://breast-cancer-research.com/

WHO (2014). World Cancer Report. Retrieved from http://www.iarc.fr/en/publications/books/wcr/wcr-order.php

Wrang, Yo, He, Che, Li, Yang, Shu (2013) Cognitive emotion regulation strategies as predictors of depressive symptoms in women newly diagnosed with breast cancer. Retrieved from http://www.researchgate.net/publication/256613071_Cognitive_emotion_regulation_strat egies_as_predictors_of_depressive_symptoms_in_women_newly_diagnosed_with_breast _cancer 